\title{
Efecto del uso de probióticos en la morfometría intestinal de pollos de engorde
}

\author{
Roa, M.L. ${ }^{@}$, Guzmán, Y.E. y Navarro, C.A.
}

Grupo Investigación en Agroforestería. Universidad de los Llanos. Villavicencio. Colombia.

\section{PaLABRAS CLAVE ADICIONALES}

Metabolismo

Digestibilidad.

Vellosidades intestinales.

Criptas intestinales.

Duodeno.

\section{RESUMEN}

Este trabajo se realizó en el laboratorio de histopatología y en la unidad experimental avícola de la Granja de la Universidad de los Llanos, ubicada en la vereda Barcelona de VillavicencioColombia, en el cual se evaluó el efecto del uso de probióticos sobre los parámetros morfométricos en pollos de engorde suplementados con dietas balanceadas remplazando la proteína en cuatro niveles de sustitución $(0,5,10$ y $15 \%)$ con harina de botón de oro, con y sin adición de probióticos (Saccharomyces cerevisiae, Lactobacillus sp. y Bacillus sp.), mediante la determinación de la altura y amplitud de las vellosidades, y profundidad y amplitud en criptas del duodeno, yeyuno e íleon. Se observó una diferencia entre los tratamientos $(\mathrm{P}<0,05)$ en los cuales se utilizaron probióticos, pre sentando un mejor resultado el tratamiento con adición de Bacillus subtilis con sustitución del $5 \%$ de proteína con harina de Tithonia diversifolia, donde las vellosidades duodenales presentaron mayor altura, lo cual fisiológicamente permite una mejor absorción de nutrientes al aumentar su superficie, reflejado en los parámetros zootécnicos del estudio. Se concluye que el uso de los probióticos administrados de forma individual o en mezcla para la alimentación de pollos de engorde tiene un efecto positivo sobre la morfometría intestinal, ayudando de esta manera a mejorar la absorción de nutrientes en el tracto gastrointestinal de las aves, lo cual se ve reflejado de forma positiva en los parámetros zootécnicos de interés para el avicultor, tales como: conversión alimenticia, ganancia de peso diaria, ganancia de peso total e índices de mortalidad.

\section{Effect of using probiotics in the intestinal morphometry of broiler}

\section{SUMMARY}

\section{ADDITIONAL KEYWORDS}

Metabolism.

Digestibility.

Intestinal villi.

Intestinal crypts.

Duodenum.

\section{INFORMATION}

Cronología del artículo.

Recibido/Received: 23.12.2016

Aceptado/Accepted: 01.09.2018

On-line: 15.10 .2018

Correspondencia a los autores/Contact e-mail:

mroa@unillanos.edu.co

\section{INTRODUCCIÓN}

El crecimiento constante de la población humanal, ha obligado al sector agropecuario a aumentar los niveles de producción de proteína de origen animal en poco tiempo, cumpliendo con la exigencia de brindar una excelente calidad e inocuidad en los productos finales, de tal forma que permita asegurar el abastecimiento de
This work was done in the laboratory of Histopathology and in the poultry experimental unit Farm University of the Llanos, located in the Barcelona village of Villavicencio-Colombia, in which was evaluated the effect of the use of probiotics on the morphometric parameters in broilers supplemented with balanced diets, replacing the protein at four levels of substitution $(0,5,10$ and $15 \%)$ with flour buttercup, with and without addition of probiotics (Saccharomyces cerevisiae, Lactobacillus sp. y Bacillus sp.), through the determination of height and width of the villi, and depth and amplitude in crypts of the duodenum, jejunum and ileum. A difference was observed between treatments $(P<0.05)$ in which probiotics were used, presenting a better result the treatment with addition of Bacillus subtilis with substitution of $5 \%$ of protein with Tithonia diversifolia flour, where the duodenal villi presented higher height, which physiologically allows a better absorption of nutrients by increasing its surface, reflected in the zootechnical parameters of the study. It is concluded that the use of probiotics administered individually or in mixture for the feeding of broilers has a positive effect, which is reflected in the intestinal morphometric, helping in this way to improve the absorption of nutrients in the gastrointestinal tract of birds, which is reflected positively in the zootechnical parameters of interest to the poultry farmer, such as: feed conversion, daily weight gain, total weight gain, and mortality rates. alimento para la población. Esta alta demanda conlleva a la implementación de sistemas productivos intensivos, que sin alterar el estado fisiológico normal de los animales permiten mejorar los índices de producción sin afectar negativamente la salud pública.

En la producción pecuaria uno de los sectores más exigidos es la avicultura, que por ser una producción animal intensiva de rápida rotación, aporta productos 
alimenticios inocuos de alta calidad para suplir la creciente demanda de alimento en el mundo (P Wigley 2013, pp. 413-7). Dados los riesgos sanitarios y productivos intrínsecos de la avicultura, anteriormente se permitía el uso de antibióticos como promotores de crecimiento (APC), los cuales son reevaluados por el riesgo que generan en la salud humana (P Agostini et al. 2012, pp. 113-8; G Huyghebaert et al. 2011, pp. 182-8), por lo tanto surge el uso de probióticos como una alternativa al uso de antibióticos (APC) en la producción animal (V Ravindran 2013, pp. 62-6). Los microorganismos eficientes (ME) son un conjunto de bacterias benéficas relativamente novedosas, que presentan una gran variedad de usos, dentro de los cuales está la adición en las dietas nutricionales, principalmente en pollos de engorde, porque los resultados se pueden apreciar en un tiempo corto debido a su ciclo productivo; como en el estudio realizado por ERL Pelicano et al. (2005, pp. 169-75) en donde se evalúo el efecto de diferentes probióticos sobre la morfometría de la mucosa intestinal, encontrándose cambios respecto a la altura y perímetro de las vellosidades en duodeno.

En cuanto a las bondades de estos microorganismos se encuentra el estudio realizado por Y García et al. (2005, pp. 129-40) en el que se demostró que el uso de ME mejora el balance microbiano del tejido gastrointestinal (TGI), estimula la producción de enzimas hidrolíticas y bacterias acido lácticas favoreciendo la acidez del TGI, todo lo cual conlleva a mejorar el rendimiento productivo de las aves. M Cuervo et al. (2002, pp. 319-29) citando a otros autores, manifiestan que al suministrar un producto que proporcione los nutrientes necesarios para los pollos resulta en una variedad de efectos positivos como el mayor desarrollo de los órganos digestivos, en el caso del intestino, un aumento de la longitud de las vellosidades del mismo, lo que a su vez produce una mejor utilización de los nutrientes.

La región de los Llanos Orientales se vislumbra como la despensa de alimentos para Colombia y el mundo, en donde una de las necesidades es aumentar la producción de aves de engorde para abastecer la región y el país, lo cual reta a los productores para hacer uso de aditivos alimentarios que sostengan la ganancia de peso y el estado sanitario de los animales (F Ingrao et al. 2013, pp. 429-38); uno de ellos ha sido el uso de antibióticos como promotores de crecimiento, los cuales están en entre dicho en términos de salud pública al dejar residuos en la carne de pollo que va para consumo humano lo cual favorece la resistencia bacteriana.

En el departamento del Meta en los últimos años se han establecido nuevas producciones avícolas con el fin de abastecer el mercado no sólo regional sino el mercado central del país, obligando a los productores a aumentar el número de animales por encasetamiento o a buscar un mecanismo viable que disminuya el tiempo requerido para engordar los pollos. En esta búsqueda de nuevas opciones surge el uso de los probióticos, consolidándose como una de las alternativas naturales al uso de antibióticos promotores de crecimiento en los animales a nivel mundial, con la ventaja de no generar efectos colaterales en el animal pero especialmente en el consumidor final; también se les atribuye el mejo- ramiento de la digestibilidad, la ganancia de peso y aumento en el índice de conversión alimenticia, lo cual al final del ciclo productivo se refleja en mayor rentabilidad para el productor. Por lo anteriormente planteado, con el presente trabajo de investigación se pretende aportar a la comunidad científica y a los productores, información que pueda mejorar el rendimiento de las producciones mediante el uso de microorganismos probióticos adicionados en forma individual o en mezcla con la sustitución de un porcentaje de proteína en la dieta de los pollos de engorde, midiendo el efecto especialmente en la histología del tracto digestivo como un posible cambio de la morfometría intestinal.

Basados en los resultados de investigaciones anteriores, se pretende evaluar el efecto del uso de probióticos en la alimentación de los pollos de engorde sobre la morfometría del intestino con el objetivo de mejorar la absorción de nutrientes y por ende la conversión alimenticia, la ganancia de peso diaria, y asimismo disminuir los costos de producción.

\section{MATERIAL Y MÉTODOS}

El proyecto se realizó en la unidad experimental avícola de la Granja de la Universidad de los Llanos, ubicada en la vereda Barcelona de Villavicencio-Meta, temperatura promedio del lugar: $28^{\circ} \mathrm{C}$ y humedad relativa promedio del $85 \%$. Se utilizaron 300 pollitos raza Ross de un día de nacidos; manejados mediante protocolos zootécnicos y control sanitario habitual, con disponibilidad de agua a voluntad, alimento suplementado en comederos y manejo de cortinas, entre otros, en un diseño experimental completamente al azar con arreglo factorial $4 \times 5 \times 3 \times 5$, cuatro niveles de sustitución de torta de soya $(0,5,10$ y 15\%), cinco formas de adición de probióticos: cero, Saccharomyces cerevisiae, Lactobacillus acidophilus, Bacillus subtilis y la mezcla de los tres probióticos, y tres repeticiones simultáneas de 5 pollitos cada una, para un total de 15 animales por tratamiento, los cuales se muestran de manera resumida en la Tabla I. El procesamiento de muestras y la evaluación de la morfometría intestinal se realizaron en el laboratorio de Histopatología de la Universidad de los Llanos.

A los pollitos se les brindó calefacción y alimento comercial de pre-iniciación hasta los 15 días de edad, luego se alojaron en jaulas metálicas de tres niveles, y

Tabla I. Tratamientos con cuatro niveles de sustitución y cinco formas de adición de probiótico. (Treatments with four levels of substitution and five forms of probiotic addition)

\begin{tabular}{lcccc}
\hline \multirow{2}{*}{ Probiótico } & \multicolumn{5}{c}{$\begin{array}{c}\text { Niveles de sustitución de harina } \\
\text { de soya por Thitonia diversifolia }\end{array}$} \\
\cline { 2 - 6 } & $0 \%$ & $5 \%$ & $10 \%$ & $\mathbf{1 5 \%}$ \\
\hline Ninguno & $\mathrm{T}_{1}$ & $\mathrm{~T}_{2}$ & $\mathrm{~T}_{3}$ & $\mathrm{~T}_{4}$ \\
Saccharomyces cerevisiae $(S C)$ & $\mathrm{T}_{5}$ & $\mathrm{~T}_{6}$ & $\mathrm{~T}_{7}$ & $\mathrm{~T}_{8}$ \\
Lactobacillus acidophilus $(L A)$ & $\mathrm{T}_{9}$ & $\mathrm{~T}_{10}$ & $\mathrm{~T}_{11}$ & $\mathrm{~T}_{12}$ \\
Bacillus subtilis $(B S)$ & $\mathrm{T}_{13}$ & $\mathrm{~T}_{14}$ & $\mathrm{~T}_{15}$ & $\mathrm{~T}_{16}$ \\
$(S C)+(L A)+(B S)$ & $\mathrm{T}_{17}$ & $\mathrm{~T}_{18}$ & $\mathrm{~T}_{19}$ & $\mathrm{~T}_{20}$ \\
\hline
\end{tabular}


Tabla II. Longitud $(\mu \mathrm{m})$ de vellosidades en duodeno, yeyuno e íleon de los diferentes tratamientos. (Length $(\mu \mathrm{m})$ of villousness in the duodenum, jejunum and ileum of different treatments)

\begin{tabular}{|c|c|c|c|c|c|}
\hline \multirow{2}{*}{ Probióticos } & & \multicolumn{4}{|c|}{ Niveles de sustitución de harina de soya por Thitonia diversifolia } \\
\hline & & $0 \%$ & $5 \%$ & $10 \%$ & $15 \%$ \\
\hline \multirow{5}{*}{ Duodeno } & Ninguno & $882,7^{\mathrm{aA}}$ & $989,1^{\text {Aa }}$ & $996,4^{\text {Aa }}$ & $1068,2^{\mathrm{abA}}$ \\
\hline & SC & $1269,3^{\mathrm{bA}}$ & $1022^{\mathrm{aA}}$ & $1461^{\mathrm{cB}}$ & $1321^{\mathrm{bB}}$ \\
\hline & LA & $1507,5^{\mathrm{abB}}$ & $1188,7^{\mathrm{aA}}$ & $1212^{\mathrm{aA}}$ & $1243,5^{\mathrm{aB}}$ \\
\hline & BS & $1022,4^{\mathrm{abB}}$ & $1566,9^{\mathrm{abB}}$ & $1237^{\mathrm{bA}}$ & $1317^{\mathrm{bB}}$ \\
\hline & $S C+L A+B S$ & $1081^{\mathrm{aA}}$ & $1656^{\mathrm{bB}}$ & $1525^{\mathrm{bB}}$ & $1215^{\mathrm{aA}}$ \\
\hline \multirow{5}{*}{ Yeyuno } & Ninguno & $851,8^{\mathrm{aA}}$ & $947,6^{\mathrm{abA}}$ & $931,7^{\mathrm{abA}}$ & $932,6^{\mathrm{abA}}$ \\
\hline & SC & $1091,2^{\mathrm{abcA}}$ & $1029,5^{\text {арАв }}$ & $1146,3^{\mathrm{abc} A \mathrm{~B}}$ & $1034^{\mathrm{abc} A B}$ \\
\hline & LA & $1173,7^{\mathrm{bcc}}$ & $1063,9^{\mathrm{abcAB}}$ & $1245,8^{\mathrm{bcBC}}$ & $1084,9^{\mathrm{abc} A \mathrm{~B}}$ \\
\hline & BS & $1144,1^{\mathrm{abc} A \mathrm{~B}}$ & $1298^{\mathrm{cC}}$ & $1033,5^{\mathrm{abc} A \mathrm{~B}}$ & $1185,7^{\mathrm{bcBC}}$ \\
\hline & $S C+L A+B S$ & $1003,6^{\mathrm{abA}}$ & $1351,2^{\mathrm{cC}}$ & $999,3^{\mathrm{abAB}}$ & $1190,2^{\mathrm{bcBC}}$ \\
\hline \multirow{5}{*}{ Íleon } & Ninguno & $649,5^{\mathrm{abA}}$ & $644,2^{\mathrm{aA}}$ & $789,9^{\mathrm{abc} A \mathrm{~B}}$ & $811,4^{\mathrm{abcA}}$ \\
\hline & SC & $773,9^{\mathrm{abcAB}}$ & $799,7^{\mathrm{abcAB}}$ & $886,4^{\mathrm{abcAB}}$ & $845,8^{\mathrm{abcA}}$ \\
\hline & LA & $783,3^{\mathrm{abc} A \mathrm{~B}}$ & $774,6^{\mathrm{abc} A \mathrm{~B}}$ & $899,7^{\mathrm{bcBC}}$ & $879,7^{\mathrm{abcA}}$ \\
\hline & BS & $825,9^{\mathrm{abc} A \mathrm{~B}}$ & $924,6^{\mathrm{cc}}$ & $776^{\mathrm{abcAB}}$ & $921,9^{\mathrm{abcB}}$ \\
\hline & $S C+L A+B S$ & $758,7^{\mathrm{abc} A B}$ & $947,5^{\mathrm{cc}}$ & $699,7^{\text {abcAB }}$ & $822,9^{\mathrm{abcA}}$ \\
\hline
\end{tabular}

Filas con diferentes letras minúsculas son distintas $(P<0,05)$. Columnas con diferentes letras mayúsculas son distintas $(P<0,05)$. $S C=$ Saccharomyces cerevisiae. LA = Lactobacillus acidophilus. BS $=$ Bacillus subtilis.

fueron divididos en los 20 tratamientos dietarios, asegurando la concentración de $10^{7}$ ufc/g de cada probiótico en las dietas experimentales, las cuales se prepararon en la granja Barcelona y fueron balanceadas al 21\% de proteína y a $3000 \mathrm{Kcal} / \mathrm{K}$ de alimento (isoproteícas e isoenergéticas), con núcleo vitamínico y mineral. A las formulaciones se les hicieron análisis nutricionales antes de iniciar la etapa experimental; y se utilizaron como materias primas: Torta de soya, harina de botón de oro, salvado de trigo, torta de palma africana, sorgo y melaza.

A los 44 días de vida de los pollos, se sacrificaron dos aves por tratamiento tomados al azar, para un total de 40 aves, a los cuales se les realizó necropsia de acuerdo al protocolo establecido en el Laboratorio de Histopatología del Programa de Medicina Veterinaria y Zootecnia, obteniendo muestras de tejido intestinal de aproximadamente $2 \mathrm{~cm}$, extrayendo duodeno de la parte proximal en la porción media del páncreas; yeyuno de la porción posterior del vestigio del saco vitelino, e íleon en la porción inmediatamente anterior a los sacos ciegos. Se realizaron 3 cortes de forma tubular, fijándolo las muestras con formalina bufferada al 10\%, para posteriormente realizar las placas histológicas (Figura 1A), donde se realizaron los cortes para incluirlos en los cassettes debidamente identificados y rotulados y colocarlos en el Histotecnicón durante 18 horas. Las muestras se incluyeron en parafina fundida a $60^{\circ} \mathrm{C}$ en los moldes para solidificarlas y poder realizar los cortes en el micrótomo. Los cortes se montaron en láminas de vidrio, y se introdujeron en horno a $60^{\circ} \mathrm{C}$ para retirar el exceso de parafina y realizar la coloración con hematoxilina y eosina (Figura 1B).

La medida de las vellosidades intestinales (altura y longitud) y de las criptas intestinales (profundidad y amplitud) se realizó en un microscopio óptico marca Leyca, con objetivo 4X (Figuras 1C y 1D) utilizando el programa ImageJ para tal fin, en cada lámina se observaron cuatro campos, y en cada vellosidad se tomaron dos medidas, una en la parte media y otra en el ápice. La medida de las criptas se realizó de igual forma en la parte media para su espesor; posteriormente se tabularon los datos en el programa Excel Microsoft 2010, para su posterior análisis de varianza (ANOVA), sometiendo los resultados a la prueba de comparación de medias Tukey con un nivel de significancia de $\mathrm{P}<0,05$ utilizando el programa estadístico SPSS versión 19.

El modelo estadístico corresponde al diseño completamente al azar con arreglo factorial, cuya fórmula es:

$$
\mathrm{Y}_{\mathrm{ijk}}=\boldsymbol{\mu}+\alpha_{\mathrm{j}}+\boldsymbol{\beta}_{\mathrm{k}}+\alpha \boldsymbol{\beta}_{j \mathrm{k}}+\boldsymbol{\varepsilon}_{\mathrm{ijk}}
$$

En donde: $Y_{\mathrm{ijk}}=$ Variable respuesta de la puntuación del $i$ sujeto bajo la combinación del $j$ valor del factor A y el $k$ valor del factor B. $\mu=$ Efecto de la media general. $\boldsymbol{\alpha}_{j}=$ Efecto de $j$ nivel de la variable de tratamiento A (cuatro niveles de reemplazo de la proteína). $\boldsymbol{\beta}_{k}=$ Efecto del $k$ valor de la variable de tratamiento B (cinco tratamientos sin y con probióticos). $\boldsymbol{\alpha} \boldsymbol{\beta}_{j k}=$ Efecto de la interacción entre el $i$ valor de A y el $k$ valor de B. $\varepsilon_{i j}$ $=$ Error experimental o efecto aleatorio de muestreo..

\section{RESULTADOS Y DISCUSIÓN}

Para la variable altura de vellosidades en el duodeno se pueden observar diferencias estadísticamente significativas $(\mathrm{P}<0,05)$ para probióticos en $\mathrm{T}_{9} \mathrm{y}_{13}$ respecto a $\mathrm{T}_{1}$; así como para $\mathrm{T}_{14} \mathrm{y} \mathrm{T}_{18}$ respecto a $\mathrm{T}_{2 ;}$ de igual manera se observaron diferencias en $\mathrm{T}_{7}$ y $\mathrm{T}_{19}$ respecto a $T_{3}, y$ en $T_{8}, T_{12}, T_{16}$ respecto al tratamiento control $\left(T_{4}\right)$. Entre los niveles de sustitución de proteína con botón 
de oro, presentaron diferencias significativas $(\mathrm{P}<0,05)$ $\mathrm{T}_{7}, \mathrm{~T}_{12}, \mathrm{~T}_{14} \mathrm{y} \mathrm{T}_{18}$. Mientras que la porción intestinal duodeno presentó los mayores valores de medidas de las vellosidades intestinales (altura y longitud) y criptas intestinales (profundidad y amplitud) en $\mathrm{T}_{14} \mathrm{y} \mathrm{T}_{18}$ correspondientes a la dieta con el $5 \%$ de sustitución de proteína y la inclusión de Bacillus subtilis, y a la mezcla de los tres probióticos respectivamente. Por otro lado, en la longitud de las vellosidades del yeyuno se observó diferencias estadísticamente significativas en $\mathrm{T}_{14} \mathrm{y}$ $\mathrm{T}_{18}$, siendo este último el qué presentó los valores más altos para la variable. $\mathrm{T}_{16} \mathrm{y} \mathrm{T}_{20}$ presentaron diferencias significativas $(\mathrm{P}<0,05)$ respecto al tratamiento control: $\mathrm{T}_{4}$. En cuanto a la sustitución proteica se pudo observar una diferencia significativa $(\mathrm{P}<0,05)$ entre $\mathrm{T}_{18} \mathrm{y} \mathrm{T}_{17}$. Por otro lado en el íleon se observan vellosidades de menor tamaño que en las otras secciones intestinales donde se siguen observando los mejores resultados con diferencias significativas $(\mathrm{P}<0,05)$ en $\mathrm{T}_{14} \mathrm{y} \mathrm{T}_{18}$, tanto para la inclusión de probióticos como para la sustitución proteica, y también se halló una diferencia significativa $(\mathrm{P}<0,05)$ en $\mathrm{T}_{16}$ con respecto a $\mathrm{T}_{4}$ en la inclusión de Bacillus subtilis con el 15\% de sustitución de proteína (Tabla II).

Para la variable longitud de las vellosidades del duodeno se observaron diferencias significativas $(\mathrm{P}<0,05)$ en cuanto a la inclusión de probióticos en $\mathrm{T}_{13}$ $\mathrm{y}_{14}$ correspondientes a Bacillus subtilis, $\mathrm{T}_{8}$ que pertenece al grupo de dietas con adición de Saccharomyces cerevisiae presentó los mejores resultados en cuanto a la variable, $\mathrm{y}_{19}$ correspondiente a la mezcla de los tres probióticos. En cuanto a niveles de sustitución de proteína se observan diferencias significativas $(\mathrm{P}<0,05)$ en $\mathrm{T}_{8} \mathrm{y} \mathrm{T}_{14}$ que corresponden al 15 y $5 \%$ de sustitución de proteína con harina de botón de oro respectivamente. Así mismo en el yeyuno se observaron diferencias significativas $(\mathrm{P}<0,05)$ para la inclusión de probióticos en $\mathrm{T}_{14}, \mathrm{~T}_{18} \mathrm{y} \mathrm{T}_{20}$, correspondientes a Bacillus subtilis y a la mezcla de los tres probióticos; en los niveles de sustitución de proteína se observó diferencia significativa $(\mathrm{P}<0,05)$ en $\mathrm{T}_{20} \mathrm{y} \mathrm{T}_{19}(15$ y $10 \%$ de sustitución proteica respectivamente). Mientras que en el íleon no se observó una diferencia significativa $(\mathrm{P}>0,05)$ entre el control con el $0 \%$ de inclusión de proteína y sin probióticos y los demás tratamientos, pero si $(\mathrm{P}<0,05)$ entre $\mathrm{T}_{5} \mathrm{y}$ $\mathrm{T}_{9}$, y entre $\mathrm{T}_{5} \mathrm{y} \mathrm{T}_{13}$ que corresponden a Saccharomyces cerevisiae con los valores más bajos, Lactobacillus acidophilus y Bacillos subtilis, respectivamente. $\mathrm{T}_{14}$ mostró una diferencia significativa $(\mathrm{P}<0,05)$ con respecto a $T_{2}$ para la inclusión de probióticos, y con $\mathrm{T}_{13} \mathrm{y} \mathrm{T}_{15}$ para la sustitución proteica. De igual manera $\mathrm{T}_{16} \mathrm{y} \mathrm{T}_{20}$ fueron diferentes mostrando significancia $(\mathrm{P}<0,05)$ con respecto a $\mathrm{T}_{4}$ (Tabla III).

De acuerdo a los resultados obtenidos durante la investigación se pudo observar una diferencia entre los tratamientos puesto que las medidas para altura y longitud de vellosidades en los cuales se utilizaron probióticos fueron mayores $(\mathrm{P}<0,05)$, presentando un mejor resultado el tratamiento con adición de Bacillus subtilis con una sustitución del 5\% de proteína con harina de botón de oro (Tithonia diversifolia) donde las vellosidades duodenales presentaron mayor altura, seguido por el tratamiento en el cual se utilizó la mezcla de los 3 probióticos; lo cual fisiológicamente permite un mayor aprovechamiento de nutrientes al aumentar la superficie de absorción, reflejado en los parámetros zootécnicos del estudio; coincidiendo con lo reportado por I Pinto y S Rodríguez (2012, pp. n.d) quienes realizaron un ensayo para evaluar el efecto de la adición en el agua de bebida del probiótico "Lactobac®" en el desarrollo alométrico de las vellosidades duodenales y los parámetros zootécnicos en pollos de

Tabla III. Amplitud $(\mu \mathrm{m})$ de vellosidades en duodeno, yeyuno e íleon de los diferentes tratamientos (Amplitude $(\mu \mathrm{m})$ of villousness in the duodenum, jejunum and ileum of different treatments)

\begin{tabular}{|c|c|c|c|c|c|}
\hline \multirow{2}{*}{ Probióticos } & & \multicolumn{4}{|c|}{ Niveles de sustitución de harina de soya por Thitonia diversifolia } \\
\hline & & $0 \%$ & $5 \%$ & $10 \%$ & $15 \%$ \\
\hline \multirow{5}{*}{ Duodeno } & Ninguno & $65,6^{\mathrm{aA}}$ & $81,2^{\mathrm{aA}}$ & $79,5^{\mathrm{aA}}$ & $98,7^{\mathrm{abAB}}$ \\
\hline & $\mathrm{SC}$ & $89,6^{\mathrm{aA}}$ & $95,5^{\mathrm{abA}}$ & $110,8^{\mathrm{abc} A \mathrm{~B}}$ & $159,7^{\circ C}$ \\
\hline & LA & $84,1^{\mathrm{aA}}$ & $110,7^{\mathrm{abcBC}}$ & $102,2^{\mathrm{abAB}}$ & $82,5^{\mathrm{aA}}$ \\
\hline & BS & $118,3^{\mathrm{abc} A \mathrm{~B}}$ & $151,9^{\mathrm{bcc}}$ & $83,7^{\mathrm{aA}}$ & $114,6^{\mathrm{abcBC}}$ \\
\hline & $S C+L A+B S$ & $85,2^{\mathrm{aA}}$ & $150,4^{\mathrm{bcc}}$ & $115,3^{\mathrm{abcBC}}$ & $91,9^{\mathrm{aA}}$ \\
\hline \multirow{5}{*}{ Yeyuno } & Ninguno & $78^{\mathrm{abAB}}$ & $80,3^{\mathrm{abAB}}$ & $75,6^{\mathrm{abABC}}$ & $72^{\mathrm{aA}}$ \\
\hline & $\mathrm{SC}$ & $76,4^{\mathrm{abAB}}$ & $84,6^{\mathrm{ab} A \mathrm{~B}}$ & $98,9^{\mathrm{abcABC}}$ & $85,1^{\mathrm{abAB}}$ \\
\hline & LA & $71,2^{\mathrm{aA}}$ & $81,7^{\mathrm{ab} A \mathrm{~B}}$ & $88^{\mathrm{abcBC}}$ & $85,1^{\mathrm{abAB}}$ \\
\hline & BS & $86,7^{\mathrm{abc} A B C}$ & $116,5^{\mathrm{bcc}}$ & $80,9^{\mathrm{abAB}}$ & $102,1^{\mathrm{abcBC}}$ \\
\hline & $S C+L A+B S$ & $77,2^{\mathrm{abAB}}$ & $105,4^{\mathrm{bcBC}}$ & $76,8^{\mathrm{ab} A B}$ & $118,7^{\mathrm{cc}}$ \\
\hline \multirow{5}{*}{ Íleon } & Ninguno & $75,4^{\mathrm{abAB}}$ & $61,6^{\mathrm{abA}}$ & $66,6^{\mathrm{abA}}$ & $63,7^{\mathrm{abA}}$ \\
\hline & SC & $59,8^{\mathrm{aA}}$ & $81,7^{\mathrm{abc} A}$ & $75,5^{\mathrm{abA}}$ & $71,8^{\mathrm{abA}}$ \\
\hline & LA & $83,5^{\mathrm{bcBC}}$ & $75,8^{\mathrm{abA}}$ & $68,3^{\text {abA }}$ & $81,2^{\mathrm{abcA}}$ \\
\hline & BS & $76,4^{\mathrm{abB}}$ & $101,5^{\mathrm{cB}}$ & $77,6^{\mathrm{abA}}$ & $90,4^{\mathrm{bcBC}}$ \\
\hline & $S C+L A+B S$ & $68,8^{\mathrm{abAB}}$ & $69,2^{\mathrm{abA}}$ & $73,3^{\mathrm{abA}}$ & $87,1^{\mathrm{bcB}}$ \\
\hline
\end{tabular}

Filas con diferentes letras minúsculas son distintas $(P<0,05)$. Columnas con diferentes letras mayúsculas son distintas $(P<0,05)$. $S C=$ Saccharomyces cerevisiae. LA = Lactobacillus acidophilus. BS = Bacillus subtilis. 

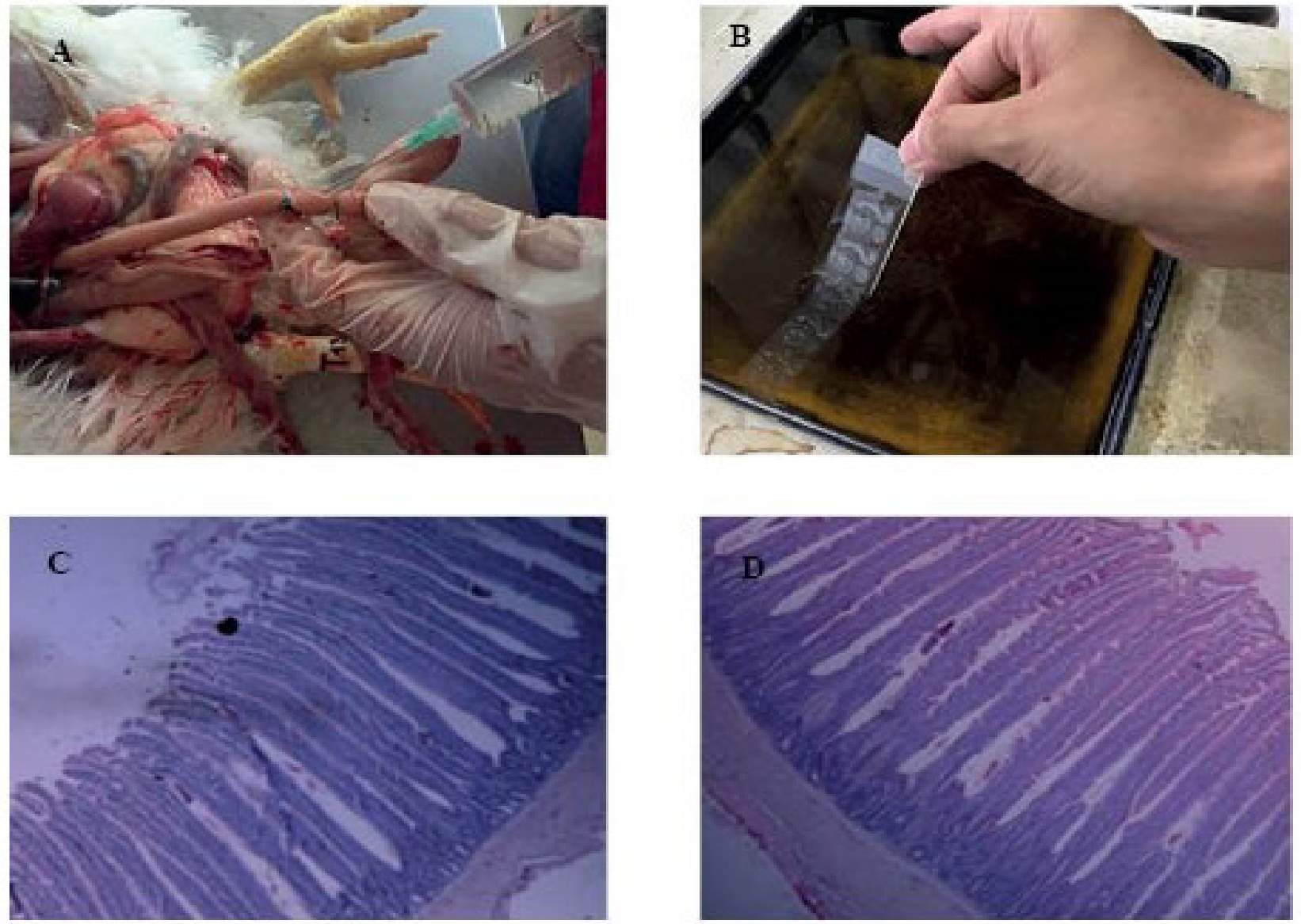

Figura 1. Obtención de muestras de intestino y montaje de palcas histológicas. (Obtaining intestinal samples and assembling histological plaques). A. Obtención de muestras del intestino de los animales bajo tratamiento. (Obtaining sam-ples from the intestine of animals under treatment). B. Tinción de placas histológicas con hematoxilina-eosina. (Staining of histological plaques with hematoxylin-eosin). C y D. Vel-losidades y criptas intestinales. Aumento 4X. (Villousness and intestinal crypts. 4X Increase).

Tabla IV. Profundidad $(\mu \mathrm{m})$ de criptas en duodeno, yeyuno e íleon de los diferentes tratamientos (Depth $(\mu \mathrm{m})$ of crypts in the duodenum, jejunum and ileum of different treatments)

\begin{tabular}{|c|c|c|c|c|c|}
\hline \multirow{2}{*}{ Probióticos } & & \multicolumn{4}{|c|}{ Niveles de sustitución de harina de soya por Thitonia diversifolia } \\
\hline & & $0 \%$ & $5 \%$ & $10 \%$ & $15 \%$ \\
\hline \multirow{5}{*}{ Duodeno } & Ninguno & $305,1^{\mathrm{CC}}$ & $285,5^{\mathrm{bcB}}$ & $267^{\mathrm{abcBC}}$ & $272,5^{\mathrm{bcBC}}$ \\
\hline & $\mathrm{SC}$ & $302,6^{\mathrm{cC}}$ & $237,6^{\mathrm{abc} A B}$ & $287,2^{\mathrm{bcBC}}$ & $217^{\mathrm{abc} A B}$ \\
\hline & LA & $191,3^{\text {abca }}$ & $202,8^{\mathrm{abc} A \mathrm{~B}}$ & $249,5^{\mathrm{abcAB}}$ & $251,1^{\mathrm{abcABC}}$ \\
\hline & BS & $154,5^{\mathrm{aA}}$ & $158,3^{\mathrm{aA}}$ & $186,4^{\mathrm{abAB}}$ & $223,1^{\mathrm{abcAB}}$ \\
\hline & $S C+L A+B S$ & $253,4^{\mathrm{abcA}}$ & $169,4^{\mathrm{abA}}$ & $243,2^{\mathrm{abcAB}}$ & $215,4^{\mathrm{abc} A B}$ \\
\hline \multirow{5}{*}{ Yeyuno } & Ninguno & $330,8^{c c}$ & $233,1^{\mathrm{abc} A B}$ & $233^{\mathrm{abc} A \mathrm{~B}}$ & $279^{\mathrm{bc} A B}$ \\
\hline & SC & $283,2^{\mathrm{bcBC}}$ & $224,3^{\mathrm{abc} A B}$ & $248,7^{\mathrm{abc} A \mathrm{~B}}$ & $314,2^{\mathrm{bcBC}}$ \\
\hline & LA & $182,3^{\mathrm{ab} A \mathrm{~B}}$ & $241,4^{\mathrm{abc} A B}$ & $242,8^{\mathrm{abcAB}}$ & $197,2^{\mathrm{abAB}}$ \\
\hline & BS & $256,4^{\mathrm{abcBC}}$ & $151,9^{\mathrm{aA}}$ & $198,2^{\mathrm{abAB}}$ & $242,4^{\mathrm{abc} A B}$ \\
\hline & $\mathrm{SC}+\mathrm{LA}+\mathrm{BS}$ & $158,5^{\mathrm{ab} A B}$ & $164,1^{\mathrm{ab} A B}$ & $193,5^{\mathrm{abAB}}$ & $212,9^{\mathrm{abc} A B}$ \\
\hline \multirow{5}{*}{ Íleon } & Ninguno & $194,8^{\mathrm{abA}}$ & $164,4^{\mathrm{abA}}$ & $221,7^{\mathrm{Bb}}$ & $191,1^{\mathrm{abA}}$ \\
\hline & $\mathrm{SC}$ & $200,5^{\mathrm{abA}}$ & $208,8^{\mathrm{bB}}$ & $141,2^{\mathrm{aA}}$ & $186,7^{\mathrm{abA}}$ \\
\hline & LA & $186,5^{\mathrm{abA}}$ & $197,4^{\mathrm{abA}}$ & $168,7^{\mathrm{abAB}}$ & $177,6^{\mathrm{abA}}$ \\
\hline & BS & $167,1^{\mathrm{abA}}$ & $144,2^{\mathrm{aA}}$ & $161,2^{\mathrm{abAB}}$ & $185,7^{\mathrm{abA}}$ \\
\hline & $S C+L A+B S$ & $180^{\mathrm{abA}}$ & $140,5^{\mathrm{aA}}$ & $154,4^{\mathrm{abAB}}$ & $177,6^{\mathrm{abA}}$ \\
\hline
\end{tabular}

Filas con diferentes letras minúsculas son distintas $(P<0,05)$. Columnas con diferentes letras mayúsculas son distintas $(P<0,05)$. $S C=$ Saccharomyces cerevisiae. LA = Lactobacillus acidophilus. BS = Bacillus subtilis. 
engorde, encontrando efectos benéficos en la altura (largo) y longitud (ancho) de las vellosidades duodenales en pollos de engorde ayudando en el desarrollo alométrico de las mismas, y con el estudio realizado por RS Gómez et al. (2009, pp. 1-12) donde se evaluó en pollos de engorde de raza Ross la productividad, digestibilidad ileal, retención de nutrientes y morfología intestinal en pollos de 28 a 42 días de edad alimentados con mananooligosacáridos y un cultivo de levaduras (MOS+CSc) combinados con Bacillus subtilis (BS) vivo; donde la inclusión de MOS+CSc incrementó la ganancia de peso y el rendimiento de la pechuga; la digestibilidad ileal de materia seca, cenizas y energía fue menor con la dieta sin aditivos $(\mathrm{P}<0,05)$, mientras que con la dieta adicionada con BS y la dieta adicionada con MOS+CSc y BS, la digestibilidad de estos nutrientes fue mayor $(\mathrm{P}<0,05)$. El espesor de las vellosidades fue mayor $(\mathrm{P}<0,05)$ con la dieta con MOS+CSc combinado con BS (interacción de MOS+CSc y BS).

Para la variable profundidad de criptas en duodeno se presentaron diferencias significativas $(\mathrm{P}<0,05)$ en la inclusión de probióticos en $\mathrm{T}_{9} \mathrm{~T}_{13} \mathrm{y}_{17}$ con respecto a $\mathrm{T}_{1}$; de igual manera se presentó una diferencia $(\mathrm{P}<0,05)$ en $\mathrm{T}_{14} \mathrm{y} \mathrm{T}_{18}$ con respecto a $\mathrm{T}_{2}$. En cuanto a la sustitución de proteína no se presentaron diferencias significativas entre los tratamientos $(\mathrm{P}>0,05)$. En esta misma variable en el yeyuno se observaron diferencias significativas $(\mathrm{P}<0,05)$ en $\mathrm{T}_{9}$ y $\mathrm{T}_{17}$ en comparación con $\mathrm{T}_{1}$; en cuanto a los porcentajes de sustitución proteica no se observaron diferencias significativas $(\mathrm{P}>0,05)$. Mientras que la profundidad de criptas en el íleon, presentó diferencias significativas $(\mathrm{P}<0,05)$ entre $\mathrm{T}_{6} \mathrm{y} \mathrm{T}_{2}$; y entre $\mathrm{T}_{7}$ y $\mathrm{T}_{3}$. Los valores positivos como respuesta a la adición de probióticos los presentó $\mathrm{T}_{14} \mathrm{y} \mathrm{T}_{18}$ los cuales muestran una diferencia significativa con $\mathrm{T}_{6}(\mathrm{P}<0,05)$; es decir que presentó un mejor comportamiento los tratamientos a las que se les adicionó Bacillus subtilis y la mezcla de los tres probióticos, en comparación con el Sacharomyces cerivisiae el cual presentó criptas de mayor profundidad cuando la inclusión de proteína fue del 5\% (Tabla IV).

Para la variable amplitud de criptas en duodeno se observó una diferencia significativa entre $\mathrm{T}_{13}$ y $\mathrm{T}_{1}$ $(\mathrm{P}<0,05)$; de la misma manera se observó un efecto positivo del uso Bacillus subtilis y la mezcla de los tres probióticos en $\mathrm{T}_{14} \mathrm{y} \mathrm{T}_{18}$, los cuales presentaron diferencia con $\mathrm{T}_{6}(\mathrm{P}<0,05)$. Así mismo $\mathrm{T}_{7}, \mathrm{~T}_{11} \mathrm{y} \mathrm{T}_{15}$ presentaron una diferencia significativa $(\mathrm{P}<0,05)$ en comparación con $\mathrm{T}_{3}$; al igual que $\mathrm{T}_{12}$ y $\mathrm{T}_{4}$ donde la diferencia observada se atribuyó al uso del probiótico en la dieta; las diferencias atribuidas a la sustitución de proteína se pudieron observar entre $\mathrm{T}_{1} \mathrm{y} \mathrm{T}_{3}, \mathrm{~T}_{13} \mathrm{y} \mathrm{T}_{14}, \mathrm{y} \mathrm{T}_{13} \mathrm{y} \mathrm{T}_{15}$. De manera similar, en la amplitud de criptas del yeyuno, se observan diferencias significativas $(\mathrm{P}<0,05)$ para la adición de probióticos entre $\mathrm{T}_{14} \mathrm{y} \mathrm{T}_{2}$, y entre $\mathrm{T}_{12} \mathrm{y} \mathrm{T}_{16}$. Para la sustitución de proteína se presentaron diferencias $(\mathrm{P}<0,05)$ entre $\mathrm{T}_{1} \mathrm{y} \mathrm{T}_{4}, \mathrm{~T}_{3}$ y $\mathrm{T}_{4}, \mathrm{~T}_{13} \mathrm{y} \mathrm{T}_{14, \mathrm{y}} \mathrm{T}_{14} \mathrm{y}$ $\mathrm{T}_{16}$. Mientras que en el íleon se observaron diferencias significativas $(\mathrm{P}<0,05)$ para la inclusión de probióticos entre $\mathrm{T}_{9} \mathrm{y} \mathrm{T}_{13}, \mathrm{y} \mathrm{T}_{9} \mathrm{y} \mathrm{T}_{17}$; también se observaron diferencias $(\mathrm{P}<0,05)$ entre el tratamiento control $\mathrm{T}_{2}, \mathrm{~T}_{6}, \mathrm{~T}_{10}, \mathrm{y}$ $\mathrm{T}_{14} \mathrm{y}_{18}$. En lo referente a la sustitución de proteína se presentaron diferencias significativas $(\mathrm{P}<0,05)$ entre $\mathrm{T}_{1}$ y $\mathrm{T}_{2}$; y entre $\mathrm{T}_{2} \mathrm{y} \mathrm{T}_{4}$ (Tabla V).

De acuerdo a los resultados obtenidos para profundidad y amplitud de criptas se puede observar el efecto benéfico de los probióticos en los tratamientos en los cuales se incluyó el uso de los mismos, obteniéndose entonces una disminución en estas varables permitiendo así un mayor aprovechamiento de los nutrientes, los tratamientos con mejores resultados se pueden relacionar con los resultados de todas las variables es-

Tabla V. Amplitud ( $\mu \mathrm{m})$ de criptas en duodeno, yeyuno e íleon de los diferentes tratamientos (Amplitude ( $\mu \mathrm{m})$ of crypts in the duodenum, jejunum and ileum of different treatments).

\begin{tabular}{|c|c|c|c|c|c|}
\hline \multirow{2}{*}{ Probióticos } & & \multicolumn{4}{|c|}{ Niveles de sustitución de harina de soya por Thitonia diversifolia } \\
\hline & & $0 \%$ & $5 \%$ & $10 \%$ & $15 \%$ \\
\hline \multirow{5}{*}{ Duodeno } & Ninguno & $54,6^{\mathrm{abAB}}$ & $65,2^{\mathrm{abcAB}}$ & $96^{\mathrm{cC}}$ & $72,6^{\mathrm{bcBC}}$ \\
\hline & SC & $66,7^{\mathrm{abcAB}}$ & $92,4^{\mathrm{bcc}}$ & $53,1 \mathrm{abAB}$ & $58,9^{\mathrm{abcAB}}$ \\
\hline & LA & $54,1^{\mathrm{abAB}}$ & $65,1^{\mathrm{abcAB}}$ & $62,3^{\mathrm{abcAB}}$ & $49,2^{\mathrm{abA}}$ \\
\hline & BS & $85,7^{\mathrm{bcc}}$ & $43,9^{\mathrm{aA}}$ & $32^{\mathrm{aA}}$ & $56,9^{\mathrm{abc} A \mathrm{~B}}$ \\
\hline & $S C+L A+B S$ & $58,2^{\mathrm{abcAB}}$ & $47^{\mathrm{abA}}$ & $85^{\mathrm{bcc}}$ & $63,8^{\mathrm{abcBC}}$ \\
\hline \multirow{5}{*}{ Yeyuno } & Ninguno & $60,1^{\mathrm{abA}}$ & $71,8^{\mathrm{bcB}}$ & $62,8^{\mathrm{abA}}$ & $85,8^{\mathrm{cc}}$ \\
\hline & $\mathrm{SC}$ & $60,6^{\mathrm{abA}}$ & $51,1^{\mathrm{abAB}}$ & $64,3^{\mathrm{abcA}}$ & $52,4^{\mathrm{abAB}}$ \\
\hline & LA & $57,9^{\mathrm{abA}}$ & $50,1^{\mathrm{abAB}}$ & $57,6^{\mathrm{abA}}$ & $48,4^{\mathrm{abA}}$ \\
\hline & BS & $73,1^{\mathrm{bcB}}$ & $45,1^{\mathrm{Aa}}$ & $56,3^{\mathrm{abA}}$ & $72,4^{\mathrm{bcBC}}$ \\
\hline & $S C+L A+B S$ & $59,3^{\mathrm{abA}}$ & $51,2^{\mathrm{abAB}}$ & $50,4^{\mathrm{abA}}$ & $50,8^{a b A B}$ \\
\hline \multirow{5}{*}{ Íleon } & Ninguno & $50,1^{\mathrm{bAB}}$ & $62,2^{\mathrm{cc}}$ & $51,1^{\mathrm{bcBC}}$ & $47,7^{\mathrm{abA}}$ \\
\hline & SC & $48,2^{\mathrm{abAB}}$ & $47,6^{\mathrm{abAB}}$ & $58,9^{\mathrm{bcBC}}$ & $50,3^{\mathrm{bA}}$ \\
\hline & LA & $55,5^{\mathrm{bcB}}$ & $42,9^{\mathrm{abAB}}$ & $58,4^{\mathrm{bcBC}}$ & $47,5^{\mathrm{abA}}$ \\
\hline & BS & $42,8^{\mathrm{abA}}$ & $38,4^{\mathrm{Aa}}$ & $48^{\mathrm{abAB}}$ & $44,7^{\mathrm{abA}}$ \\
\hline & $S C+L A+B S$ & $44^{\mathrm{abA}}$ & $38,6^{\mathrm{aA}}$ & $47,3^{\mathrm{abAB}}$ & $47,9^{\mathrm{abA}}$ \\
\hline
\end{tabular}

Filas con diferentes letras minúsculas son distintas $(P<0,05)$. Columnas con diferentes letras mayúsculas son distintas $(P<0,05)$. $S C=$ Saccharomyces cerevisiae. $\mathrm{LA}=$ Lactobacillus acidophilus. BS = Bacillus subtilis. 
tudiadas, los cuales son: $\mathrm{T}_{14} \mathrm{y}^{\mathrm{T}_{18}}$, ambos con un nivel de sustitución de proteína del 5\% y adición de Bacillus subtilis y la mezcla de los tres probióticos. En pollos, la altura de las vellosidades intestinales aumenta y la profundidad de las criptas disminuye rápidamente tras la eclosión, lo cual incrementa la superficie de absorción de nutrientes. Las aves alimentadas con la dieta basal presentaron criptas más profundas y más anchas, lo que implica un mayor requerimiento por el alto recambio celular para el mantenimiento de este tejido (I Giannenas et al. 2012, pp. 31-40; H Salim et al. 2013, pp. 2084-90; Y Xiao-Yan et al. 2011, pp. 100-7). G Cao et al. (2013, pp. 2949-55) evaluaron la inclusión de E. faecium en pollos de engorde y encontraron que a nivel intestinal las vellosidades presentaron mayor altura y la profundidad de criptas fue menor $(\mathrm{P}<0,05)$, en comparación con el control, lo que está asociado al aumento en la eficiencia de crecimiento y en la superficie de absorción de nutrientes; coincidiendo con lo encontrado en este estudio.

En el yeyuno se observó un mejor comportamiento en la altura de las vellosidades con la mezcla de S. cerevisiae, Lactobacillus sp y B. subtilis, lo cual es comparable con los resultados obtenidos por L Chávez et al. (2016, pp. 51-8) quienes realizaron un estudio para observar el crecimiento y desarrollo intestinal de aves de engorde alimentadas con cepas probióticas (Lactobacillus casei, Lactobacillus acidophilus y Enterococcus faecium) en el agua de bebida; donde la inclusión de probióticos, específicamente E. faecium mejoró el peso, desarrollo y crecimiento de órganos de importancia digestiva, específicamente intestino, lo cual se vio reflejado en vellosidades con mayor altura y longitud, y criptas menos profundas $(p<0,01)$ lo que podría mejorar la absorción de nutrientes y por consiguiente la salud de los animales.

En el íleon, el mejor comportamiento lo presentó S. cerevisiae donde esta levadura obtuvo mejores resultados en altura de las vellosidades intestinales y en profundidad de las criptas ayudando así a mejorar la absorción, lo que concuerda con el estudio realizado por N Medina et al. (2015, pp. 107-16) quienes evaluaron en 210 pollos raza Ross, la morfología intestinal con o sin suministro de biomasa de levaduras, donde hallaron diferencias significativas $(\mathrm{P}<0,05)$ en altura de las vellosidades y profundidades de criptas, aunque estas variables cambiaron solo en algunos casos, en los cuales se les atribuyó efectos benéficos a la presencia de levaduras en la dieta.

\section{AGRADECIMIENTOS}

Agradecemos a la Dirección General de Investigaciones de la Universidad de los Llanos por la financiación de este proyecto de investigación.

\section{BIBLIOGRAFÍA}

Agostini, P, Solà-Oriol, D, Nofrarías, M, Barroeta, A, Gasa, J \& Manzanilla, E 2012, Role of in-feed clove supplementation on growth performance, intestinal microbiology, and morphology in broiler chicken, Livestock Science, vol. 147, no. 1, pp. 113-8.
Cao, G, Zeng, X, Chen, A, Zhou, L, Zhang, L, Xiao, Y, et al. 2013, Effects of a probiotic, Enterococcus faecium, on growth performance, intestinal morphology, immune response, and cecal microflora in broiler chickens challenged with Escherichia coli K88, Poultry Science, vol. 92, no. 11, pp. 2949-55.

Cuervo, M, Gómez, C \& Romero, H 2002, Efecto de la utilización de un suplemento nutricional hidratado en pollos de engorde recién nacidos, Revista Colombiana de Ciencias Pecuarias, vol. 15, no. 3, pp. 319-29.

Chávez, L, López, A \& Parra, J 2016, Crecimiento y desarrollo intestinal de aves de engorde alimentadas con cepas probióticas, Archivos de Zootecnia, vol. 65, no. 249, pp. 51-8.

García, Y, García, Y, López, A \& Boucourt, R 2005, Probióticos: una alternativa para mejorar el comportamiento animal, Revista Cubana de Ciencia Agrícola, vol. 39, no. 2, pp. 129-40.

Giannenas, I, Papadopoulos, E, Tsalie, E, Triantafillou, E, Henikl, S, Teichmann, K, et al. 2012, Assessment of dietary supplementation with probiotics on performance, intestinal morphology and microflora of chickens infected with Eimeria tenella, Veterinary Parasitology, vol. 188, no. 1, pp. 31-40.

Gómez, RS, Angeles, MdL, Albarrán, RE, Ávila, FD, Varela, C \& Mojica, MdC 2009, Efectos de incluir Mananooligosacáridos más un cultivo de levaduras y un microorganismo vivo en la deta de pollos de engorde, Adial Nutritición S.L, pp. 1-12.

Huyghebaert, G, Ducatelle, R \& Van Immerseel, F 2011, An update on alternatives to antimicrobial growth promoters for broilers, The Veterinary Journal, vol. 187, no. 2, pp. 182-8.

Ingrao, F, Rauw, F, Lambrecht, B \& van den Berg, T 2013, Infectious Bursal disease: a complex host-pathogen interaction, Developmental \& Comparative Immunology, vol. 41, no. 3, pp. 429-38.

Medina, N, González, C, Matute, G \& Barahona, R 2015, Intestinal morphology in broilers with or without supply of yeast biomass from fuel ethanol production, Zootecnia Tropical, vol. 33, no. 2, pp. 107-16.

Pelicano, ERL, Souza, P, Souza, H, Oba, A, Boiago, M, Zeola, N, et al. 2005, Carcass and cut yields and meat qualitative traits of broilers fed diets containing probiotics and prebiotics, Revista Brasileira de Ciência Avícola, vol. 7, no. 3, pp. 169-75.

Pinto, I \& Rodríguez, S 2012, Evaluación alométrica de las vellosidades duodenales y los parámetros zootécnicos en pollos de engorde al adicionar probiótico (Lactobac $\circledast$ ) en el agua de bebida, Tesis de Pregrado Médico Veterinario, Fundación Universitaria Juan de Castellanos, Tunja, Colombia,

Ravindran, V 2013, Disponibilidad de piensos y nutrición de aves de corral en países en desarrollo. En: FAO (Ed), Revisión del Desarrollo Avícola: Organización de las Naciones Unidas para la Alimentación y la Agricultura (FAO), pp. 62-6.

Salim, H, Kang, H, Akter, N, Kim, D, Kim, J, Kim, M, et al. 2013, Supplementation of direct-fed microbials as an alternative to antibiotic on growth performance, immune response, cecal microbial population, and ileal morphology of broiler chickens, Poultry Science, vol. 92, no. 8, pp. 2084-90.

Wigley, P 2013, Immunity to bacterial infection in the chicken, Developmental \& Comparative Immunology, vol. 41, no. 3, pp. 413-7.

Xiao-Yan, Y, Hua-Hua, Y \& Jing-Ci, Z 2011 , Increased gut absorptive capacity in rats with severe head injury after feeding with probiotics, Nutrition, vol. 27, no. 1, pp. 100-7. 